\title{
Physiological importance of different atrioventricular intervals to improved exercise performance in patients with dual chamber pacemakers
}

\author{
RICHARD J HASKELL, WILLIAM J FRENCH \\ From the Department of Medicine, University of California at Los Angeles School of Medicine, Los Angeles, \\ California; and the Department of Medicine, Division of Cardiology, Harbor-UCLA Medical Center, \\ Torrance, California, USA
}

SUMMARY To determine the importance of different atrioventricular intervals during exercise in patients with dual chamber pacemakers, seven patients with complete heart block and sinus rhythm were exercised in different pacing modes and atrioventricular intervals: $(a)$ ventricular inhibited (VVI) pacing with no synchronous atrial augmentation or rate responsiveness; $(b)$ atrial synchronous ventricular or DDD pacing with a short mean (SD) atrioventricular interval of 66 (4) ms; and (c) DDD pacing with a long atrioventricular interval of $168(12) \mathrm{ms}$. Pacing with a short or long atrioventricular interval gave similar maximum heart rates, oxygen uptake at the anaerobic threshold, end tidal pressure of carbon dioxide or oxygen pulse (a measure of stroke volume). Pacing with either a short or long atrioventricular interval produced a significantly higher oxygen consumption and anaerobic threshold and less lactate production than VVI pacing.

During exercise a short atrioventricular interval does not provide a better cardiopulmonary performance than a long atrioventricular interval.

Dual chamber pacemakers that allow synchronous atrioventricular pacing can increase resting cardiac output by as much as $29 \%$ compared with ventricular fixed rate pacemakers. ${ }^{1-3}$ This improvement in exercise capability may be attributed either to an atrioventricular interval that results in a properly timed atrial contraction before ventricular contraction or to the atrial rate responsiveness that permits the ventricular pacing rate to increase with metabolic demands. ${ }^{-6}$ It is unclear, however, whether a specific atrioventricular interval will improve haemodynamic function during exercise in patients with dual chamber pacemakers. ${ }^{7-9}$ This study was designed to measure cardiopulmonary function to determine what atrioventricular interval contributes most to improve exercise performance in patients with dual chamber pacemakers.

\section{Patients and methods}

We studied seven patients with dual chamber

Requests for reprints to Dr William J French, Division of Cardiology, Harbor-UCLA Medical Center, 1000 West Carson Street, Torrance, California 90509, USA.

Accepted for publication 9 August 1988 pacemakers (table 1). All patients were in sinus rhythm with complete heart block and none had anterograde or retrograde conduction. Patients were able to exercise on a bicycle to exhaustion or leg fatigue. Echocardiograms were performed on all patients.

PACING PROTOCOL

Each patient performed identical exercise testing in two different pacing modes and at two atrioventricular intervals:

Ventricular inhibited pacing (VVI) with the ventricular rate programmed to the sinus rate at rest. Variation in paced rate reflected different sinus rates at rest. This mode meant that there was no synchronised atrial contraction because all patients were in complete heart block and their ventricular rates did not increase with exercise.

Universal or DDD pacing with a short atrioventricular interval as suggested by von Bibra et al. ${ }^{10}$ The mean atrioventricular interval was set at $66 \mathrm{~ms}$. Patients used their own sinus rate to control the implanted pacemaker. Thus the pacemaker functioned as if it were in the VDD mode during exercise.

Universal or DDD pacing with a long atrioventricular interval of mean (SD) 168 (12) ms. This 46 
Table 1 Clinical and exercise findings in seven patients with dual chamber pacemakers

\begin{tabular}{|c|c|c|c|c|c|c|c|c|c|}
\hline Case & Sex & $\begin{array}{l}\text { Age } \\
(y r)\end{array}$ & $\begin{array}{l}\text { Weight } \\
\text { (kg) }\end{array}$ & $\begin{array}{l}\text { Cardiac } \\
\text { diagnosis }\end{array}$ & Pacemaker & $\begin{array}{l}\text { Pacemaker } \\
\text { implanted } \\
\text { (mnth) }\end{array}$ & $\begin{array}{l}\text { Short } \\
\text { AVI } \\
\text { (ms) }\end{array}$ & $\begin{array}{l}\text { Long } \\
\text { AVI } \\
\text { (ms) }\end{array}$ & $\begin{array}{l}\text { Work rate } \\
\text { increment } \\
\text { per min }(W)\end{array}$ \\
\hline $\begin{array}{l}1 \\
2 \\
3 \\
4 \\
5 \\
6 \\
7 \\
\text { Mean }\end{array}$ & $\begin{array}{l}\mathbf{M} \\
\mathbf{M} \\
\mathbf{F} \\
\mathbf{M} \\
\mathbf{M} \\
\mathbf{M} \\
\mathbf{M} \\
(\mathbf{S D})\end{array}$ & $\begin{array}{l}63 \\
49 \\
60 \\
62 \\
55 \\
41 \\
24 \\
51(14)\end{array}$ & $\begin{array}{c}65 \\
61 \\
56 \\
110 \\
105 \\
84 \\
70 \\
79(22)\end{array}$ & $\begin{array}{l}\text { IHD } \\
\text { None } \\
\text { MVR } \\
\text { IHD } \\
\text { IHD } \\
\text { AVR } \\
\text { AVR }\end{array}$ & $\begin{array}{l}\text { Pacesetter AFP } \\
\text { Pacesetter AFP } \\
\text { Pacesetter AFP } \\
\text { Cordis Gemini } \\
\text { Pacesetter AFP } \\
\text { Intermedics Cosmos } \\
\text { Pacesetter Genesis }\end{array}$ & $\begin{array}{l}16 \\
7 \\
2 \\
36 \\
18 \\
13 \\
1 \\
13(12)\end{array}$ & $\begin{array}{l}65 \\
65 \\
65 \\
75 \\
65 \\
65 \\
65 \\
66(4)\end{array}$ & $\begin{array}{l}165 \\
165 \\
165 \\
175 \\
165 \\
150 \\
190 \\
168(12)\end{array}$ & $\begin{array}{l}5 \\
10 \\
5 \\
10 \\
10 \\
15 \\
10 \\
11(5)\end{array}$ \\
\hline
\end{tabular}

AVI, atrioventricular interval; AVR, aortic valve replacement; IHD, ischemic heart disease; MVR, mitral valve replacement.

atrioventricular interval was based on previous studies by the authors and others. ${ }^{89}$ DDD pacing allowed atrial rate responsiveness in this group of patients with sinus rhythm and resulted in VDD pacing during exercise.

\section{EXERCISE PROTOCOL}

All the exercise tests for each patient were usually performed on the same day and there was an interval of at least 30 minutes between tests. Patients were in the preselected pacing mode at least 15 minutes before the start of exercise to allow for equilibration. The different modes for each patient were selected in a random order before exercise. The patient and technician, but not the physicians, were blind to the pacing mode chosen. Informed consent was obtained from each patient and the protocol was approved by the institutional human subjects committee.

Each exercise test consisted of the patient sitting for three minutes at rest, followed by three minutes of unloaded cycling, and then incremental increases in work rate each minute. The work rate was increased by 5,10 , or $15 \mathrm{~W}$ each minute and was chosen for each patient to obtain approximately 10 minutes of exercise based on standard tables. Patients underwent maximal exercise on a bicycle until exhaustion or leg fatigue occurred. Because the purpose of this study was to assess the role of atrial augmentation during exercise, the incremental work rate and time of exercise were kept constant for each individual patient during the three exercise tests. None of the patients was being treated with $\beta$ blockers or other medications that affected sinus node function. We excluded patients who had $<15 \%$ increase in sinus rate with exercise (chronotropic incompetence) or more than minimal pulmonary disease by pulmonary function testing. No patients were in heart failure at the time of the study.

\section{EXERCISE EQUIPMENT}

The patients exercised on a calibrated, electronically braked stationary cycle ergometer (Godart, Bitthoven, Netherlands). They breathed through a rubber mouthpiece attached to a $49 \mathrm{ml}$ deadspace breathing valve (Edward Koegel, San Antonio, TX). The expiratory flow was passed over an ambient-temperature water bath through a Fleisch No. 3 pneumotachograph. A differential pressure transducer (47304A; Hewlett-Packard, Palo Alto, CA) measured pressure across the pneumotachograph to calculate expiratory flow. Gas was sampled at the mouth through a capillary tube at a rate of $60 \mathrm{ml} / \mathrm{min}$ and analysed by a mass spectrometer (MGA-1100, Perkin-Elkmer, Pomona, CA). Heart rate was continuously monitored and a three lead electrocardiogram was recorded intermittently during exercise to ensure proper pacing.

Table 2 Echocardiographic data in seven patients with dual chamber pacemakers

\begin{tabular}{|c|c|c|c|c|c|c|c|}
\hline Case & $\begin{array}{l}\text { LVIDd } \\
(\mathrm{cm})\end{array}$ & $\begin{array}{l}\text { LVIDs } \\
(\mathrm{cm})\end{array}$ & $\begin{array}{l}\text { Left } \\
\text { Atrium } \\
(\%)\end{array}$ & $\begin{array}{l}F S \\
(\%)\end{array}$ & $\begin{array}{l}E P S S \\
(\boldsymbol{m m})\end{array}$ & $\begin{array}{l}L V P W \\
(\mathrm{~cm})\end{array}$ & $\begin{array}{l}\operatorname{LVSW} W \\
(\mathrm{~cm})\end{array}$ \\
\hline $\begin{array}{l}1 \\
2 \\
3 \\
4 \\
5 \\
6 \\
7 \\
\text { Mean (SD) }\end{array}$ & $\begin{array}{l}6 \cdot 0 \\
5 \cdot 0 \\
3 \cdot 4 \\
7 \cdot 1 \\
4 \cdot 4 \\
4 \cdot 1 \\
5 \cdot 9 \\
5 \cdot 1(1 \cdot 3)\end{array}$ & $\begin{array}{l}4.7 \\
3.0 \\
2.0 \\
5.9 \\
3.6 \\
3.0 \\
3.9 \\
3.7(0.7)\end{array}$ & $\begin{array}{l}3 \cdot 8 \\
2 \cdot 9 \\
5 \cdot 2 \\
4.5 \\
4 \cdot 7 \\
4 \cdot 0 \\
3.7 \\
4 \cdot 1(0 \cdot 7)\end{array}$ & $\begin{array}{l}22 \\
40 \\
41 \\
17 \\
18 \\
29 \\
51 \\
31(13)\end{array}$ & $\begin{array}{l}C E \\
10 \\
4 \\
34 \\
14 \\
6 \\
C E \\
14(12)\end{array}$ & $\begin{array}{l}1.6 \\
1.0 \\
1.0 \\
1.6 \\
1.5 \\
1.6 \\
1.0 \\
1.3(0.3)\end{array}$ & $\begin{array}{l}1.6 \\
1.1 \\
0.8 \\
1.0 \\
1.7 \\
1.6 \\
1.0 \\
1.3(0.4)\end{array}$ \\
\hline
\end{tabular}

CE, cannot estimate; EPSS, E point septal separation; FS, fractional shortening; LVIDd, left ventricular internal dimension at end diastole; LVIDs, left ventricular internal dimension at end systole; LVPW, left ventricular posterior wall; $L V S W$, left ventricular septal wall. 
Table 3 Data (mean (SD)) on cardiopulmonary function in seven patients with dual chamber pacemakers studied during exercise

\begin{tabular}{|c|c|c|c|c|}
\hline Exercise variable & $V V I$ & $\begin{array}{l}D D D \\
\text { Short } A V I\end{array}$ & $\begin{array}{l}D D D \\
\text { Long } A V I\end{array}$ & $p^{\star}$ \\
\hline $\begin{array}{l}\text { Maximum heart rate (beats/min) } \\
\text { Maximum resp rate (min/l) } \\
\text { End tidal } \mathrm{CO}_{2}(\mathrm{~mm} \mathrm{Hg}) \\
\text { Maximum ventilation }(1 / \mathrm{min}) \\
\text { Maximum oxygen uptake (ml/kg/min) } \\
\text { Respiratory exchange ratio }(\mathrm{R}) \\
\text { Anaerobic threshold (min) } \\
\text { Oxygen uptake at anaerobic threshold }(\mathbf{l} / \mathrm{min}) \\
\text { Oxygen pulse (ml/beat) }\end{array}$ & $\begin{array}{l}78(15) \\
33(8) \\
34(7) \\
48 \cdot 3(10 \cdot 5) \\
14 \cdot 5(2 \cdot 7) \\
1 \cdot 22(0 \cdot 15) \\
8 \cdot 1(2 \cdot 0) \\
11 \cdot 4(3 \cdot 9) \\
16(10)\end{array}$ & $\begin{array}{l}123(26) \\
36(7) \\
37(8) \\
46 \cdot 9(8 \cdot 4) \\
15 \cdot 7(2 \cdot 6) \\
1 \cdot 17(0 \cdot 15) \\
9 \cdot 8(2 \cdot 8) \\
13 \cdot 0(5 \cdot 1) \\
11(6)\end{array}$ & $\begin{array}{l}128(26) \\
33(8) \\
36(6) \\
47 \cdot 0(8 \cdot 5) \\
15 \cdot 6(2 \cdot 8) \\
1 \cdot 17(0 \cdot 15) \\
10 \cdot 2(1 \cdot 6) \\
12 \cdot 8(4 \cdot 1) \\
11(5)\end{array}$ & $\begin{array}{l}<0.001 \\
\text { NS } \\
<0.05 \\
\text { NS } \\
<0.05 \\
\text { NS } \\
<0.01 \\
<0.025 \\
<0.01\end{array}$ \\
\hline
\end{tabular}

DDD, atrial synchronous ventricular pacemaker; max, maximum; VVI, ventricular inhibited pacemaker

* Statistical significance of difference between VVI and DDD modes. There were no significant differences between DDD modes with short or long atrioventricular intervals (AVI).

\section{EXERCISE VARIABLES MEASURED}

Minute ventilation, oxygen uptake, carbon dioxide output, end tidal pressure of oxygen, end tidal pressure of carbon dioxide, and the respiratory exchange ratio were measured every 30 s during rest and exercise. Oxygen pulse, calculated as oxygen uptake divided by heart rate, is an indirect measurement of stroke volume. Maximum oxygen uptake was the highest oxygen uptake measured during the exercise test. Anaerobic threshold was determined by the characteristic gas exchange results associated with the development of lactic acidosis: the point at which the ventilatory equivalent for oxygen $\left(\dot{\mathrm{V}}_{\mathrm{E}} / \dot{\mathrm{Vo}}_{2}\right)$ begins to increase while the ven-

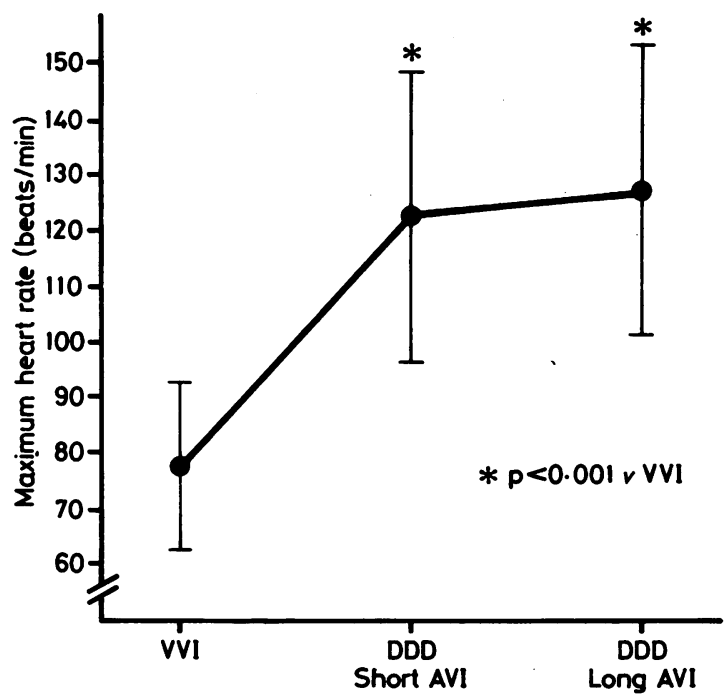

Fig 1 The maximum heart rate achieved during exercise was similar for universal or $D D D$ pacing (that is, VDD mode during exercise) with short or long atrioventricular intervals and was greater than during ventricular inhibited (VVI) pacing. AVI, atrioventricular interval. tilatory equivalent for carbon dioxide $\left(\dot{\mathrm{V}}_{\mathrm{E}} / \dot{\mathrm{VCO}}_{2}\right)$ remains constant or decreases. The physiological basis and evidence for this relation have been described elsewhere. ${ }^{11}$

\section{STATISTICAL ANALYSIS}

Variables during exercise were compared by an analysis of variance, repeated measures design. ${ }^{12}$ All values are expressed as mean (SD). A p value $<0.05$ was regarded as significant.

\section{Results}

\section{CLINICAL}

Three patients had dilated left ventricles and four patients had evidence of reduced left ventricular function as shown by the fractional shortening of the $E$ point to septal separation on the echocardiogram (table 2). There was an increase in left ventricular wall thickness in four patients consistent with left ventricular hypertrophy.

\section{CARDIOPULMONARY FUNCTION}

None of the cardiopulmonary indices measured during DDD pacing (that is VDD mode during exercise) were significantly different with short or long atrioventricular intervals ( $p=N S$, table 3$)$. Nor was there any difference in the duration of exercise during short $(12.0(2 \cdot 2) \mathrm{min})$ or long $(12.5(1.8) \mathrm{min})$ atrioventricular intervals or in the VVI mode (11.9 (2.01), $\mathrm{p}=\mathrm{NS}$ ). The maximum work rate was similar with short (61 (36) W) or long (64 (38) W) atrioventricular intervals or VVI pacing (61 (35) W, = NS).

There were important differences in several cardiopulmonary indices between pacing with short or long atrioventricular intervals and VVI pacing (table 3). As expected, there was a significant difference between maximal heart rate achieved during exercise in patients with VVI pacing and both 


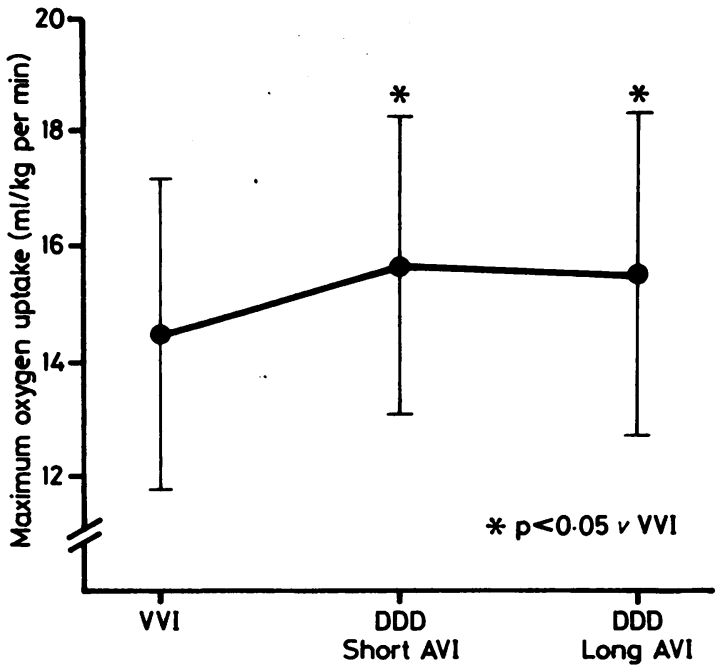

Fig 2 There was no difference in maximum oxygen uptake with either short or long atrioventricular intervals but both settings produced a higher value than ventricular inhibited (VVI) pacing. AVI, atrioventricular interval; $D D D$, universal pacing.

short or long atrioventricular intervals $(p<0.001)$ (fig 1). The maximum oxygen uptake with short or long atrioventricular intervals was significantly greater than with VVI pacing (fig 2). The end tidal pressure of carbon dioxide with short or long atrioventricular intervals was similar $(p=N S)$, but

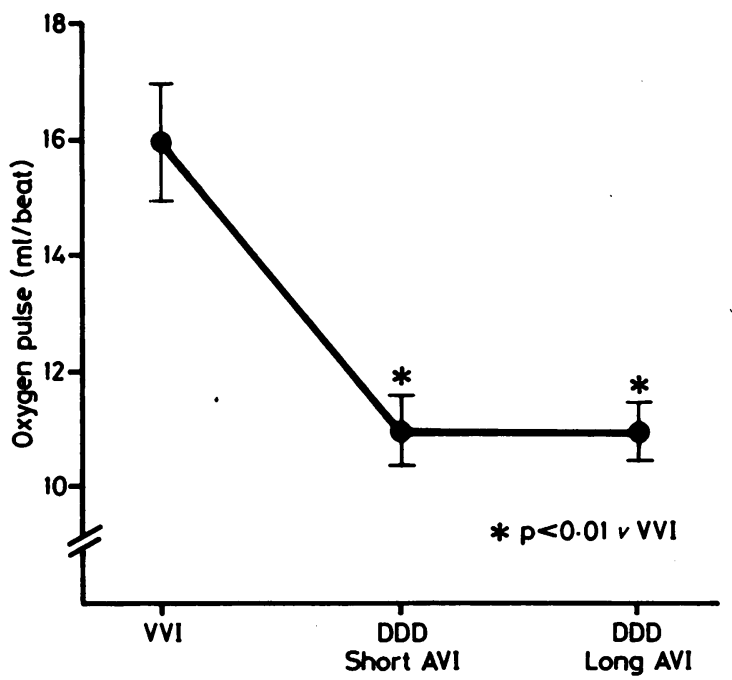

Fig 3 Patients with ventricular inhibited (VVI) pacing attained a higher oxygen pulse than patients with short or long atrioventricular intervals. AVI, atrioventricular interval; $D D D$, universal pacing.

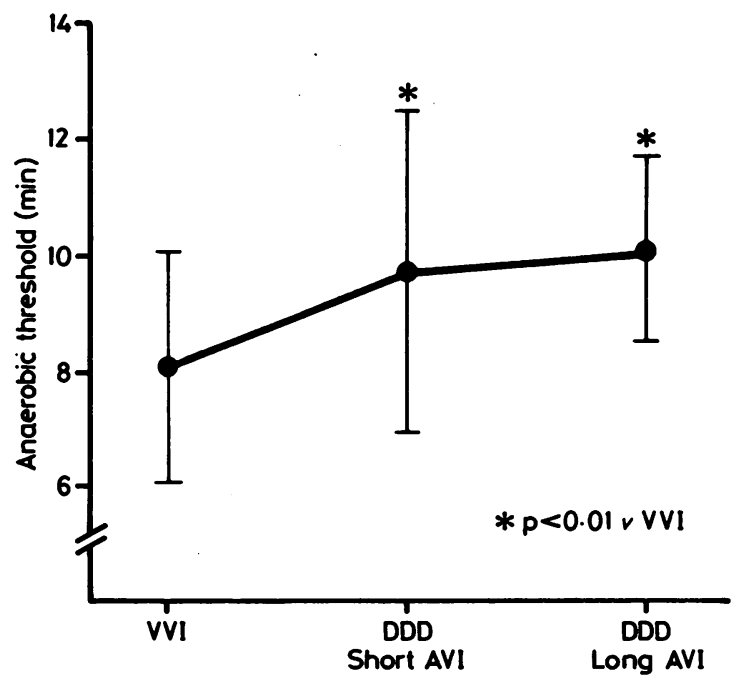

Fig 4 The anaerobic threshold was significantly higher with short or long atrioventricular intervals than with ventricular inhibited (VVI) pacing. AVI, atrioventricular interval; DDD, universal pacing.

both were higher than with VVI pacing $(p<0.05)$. The oxygen pulse (oxygen uptake/heart rate) was higher with VVI pacing than with either short or long atrioventricular intervals ( $<<0.01$, fig 3 ).

The anaerobic threshold was significantly lower with VVI pacing than with short or long atrioventricular intervals as measured by oxygen uptake at the anaerobic threshold ( $<<0.025$, table 3 ) or by the time taken to achieve anaerobic threshold (p < 0.01, fig 4). Although there were no statistically significant differences among the respiratory exchange ratios for different pacing modes there was a tendency for DDD pacing to give lower values than VVI pacing.

\section{Discussion}

In the past decade dual chamber pacemakers have been developed that permit appropriate synchronisation of atrial contraction before ventricular systole. There is little doubt that many patients benefit both clinically and haemodynamically from dual chamber pacing. ${ }^{13-15}$ The correct timing of the atrial contraction in relation to the ventricular contraction is important, because atrial augmentation may increase resting left ventricular end diastolic volume and forward cardiac output in patients with sinus rhythm by as much as $37 \% .{ }^{14-16}$ However, the usefulness of atrial synchronised contraction at higher heart rates achieved during exercise is unclear. ${ }^{6}$ Universal or DDD pacemakers can optimise the timing of atrial 
contraction by varying the atrioventricular interval and exercise performance could be maximised by an ideal setting.

Von Bibra et al studied resting $M$ mode echocardiographic measurements in patients with pacemakers and showed that a short atrioventricular interval of $50 \mathrm{~ms}$ or $75 \mathrm{~ms}$ was likely to promote more physiological conditions by maximising left ventricular filling time. ${ }^{10}$ They found that a short atrioventricular interval still produced atrial augmentation of ventricular filling (VDD) probably because of an additional $80 \mathrm{~ms}$ electrical mechanical delay between the ventricular pacing spike and the $Q$ wave on the electrocardiogram in patients with pacemakers compared with patients in sinus rhythm. Thus the functional atrioventricular delay was $80 \mathrm{~ms}$ longer than the atrioventricular interval set on the pacemaker. They concluded that, theoretically, a setting giving a short atrioventricular interval would be sufficient to maintain normal intervals between atrial and ventricular contraction in paced patients (VDD). However, Haskell and French, using pulsed Doppler derived measurements of cardiac output in resting patients showed that a short atrioventricular interval of $66 \mathrm{~ms}$ (mean) gave the lowest cardiac output. $^{8}$

Von Bibra et al also showed that a longer atrioventricular interval of $150 \mathrm{~ms}$ often meant that the mitral valve closed before the onset of ventricular systole. This would reduce late diastolic ventricular filling and presumably cardiac output. However, others showed that an atrioventricular interval of 150 or 200 ms caused the largest increase in cardiac output but than an atrioventricular interval of $250 \mathrm{~ms}$ resulted in a fall in cardiac output. ${ }^{8}$

In the current study there was no significant difference during exercise between a short and long atrioventricular interval, but both settings gave more physiological benefit than fixed rate ventricular pacing. This conclusion is based on measurement of cardiopulmonary function in patients paced in different modes during exercise. Maximum oxygen uptake was higher at both atrioventricular intervals than during VVI pacing, suggesting that both settings gave similar physiological benefits and were better than fixed rate VVI pacing during exercise. Maximum oxygen uptake is probably the best single physiological indicator of a patient's capacity for maintaining heavy work. Factors which limit maximum oxygen uptake are cardiac output, oxygen utilisation by the exercising muscles, and the gas exchange properties of the lungs. Since patients were exercised for the same amount of time and workload, oxygen use by muscles and gas exchange in the lungs were similar in all three paced groups. The increase in cardiac output probably reflected differences in atrial augmentation or heart rate or both. Values for maximal oxygen uptake were below predicted values for normal subjects because heart function was compromised. But the small changes measured in this group of patients gave an appreciable percentage change in total oxygen uptake during exercise. Most patients in this study had a reduced anaerobic threshold and reduced oxygen uptake at the anaerobic threshold. The mean values for the anaerobic threshold and oxygen uptake at the anaerobic threshold for patients with different atrioventricular intervals were similar and slightly reduced, but both variables were significantly better than in the VVI mode. Since the oxygen uptake at the anaerobic threshold defines the level of work that a patient is capable of sustaining, this suggests that the different atrioventricular settings probably permitted the patient to function at a higher workload, without resorting to anaerobic metabolism, than fixed rate VVI pacing.

End tidal carbon dioxide pressure was higher with both atrioventricular intervals; this was probably because lactate production from muscles was reduced as a result of a higher cardiac output. More lactate was likely produced with VVI pacing because cardiac output was lower. The lactate acidosis stimulates the carotid chemoreceptors and leads to hyperventilation and a lower end tidal carbon dioxide value.

Because the oxygen pulse was greatest with VVI pacing, exercise performance in this mode was probably dependent on increased oxygen extraction by the exercising muscles and not on improved cardiac output from enhanced cardiac function. Since the amount of oxygen that the muscles can extract is limited, this may account in part for the inability of patients to exercise as efficiently in the VVI mode as in either of the other two DDD settings.

\section{ATRIAL AUGMENTATION AND RATE}

\section{RESPONSIVENESS}

This study was not designed to compare the usefulness of atrial synchronised contraction with rate responsiveness. Karlof first showed that only $8 \%$ of any improvement in cardiac output could be attributed to atrioventricular synchrony in patients paced during exercise. ${ }^{1}$ Fananapazir $e t$ al found that rate, and not an appropriately timed atrial contraction, was the major physiological determinant of the $40 \%$ improvement in exercise performance in patients with rate responsive compared with fixed rate ventricular pacing. ${ }^{17}$

Shapland et al showed that in dogs with complete heart block synchronous atrical contraction only contributed a small amount to the increase in cardiac output during exercise and that a pacemaker that 
increased the rate in response to an increase in metabolic demand maintained haemodynamic values at near normal values by exercise. ${ }^{18}$

Benditt et al and Humen et al showed that in patients with an activity initiated, chronotropic-responsive pacemaker the heart was more important during exercise than it was when a single chamber fixed rate ventricular pacemaker was used. ${ }^{19} 20$ Thus it is possible that rate alone, and not a short or long atrioventricular interval, provides the greatest physiological benefit during exercise.

\section{LIMITATIONS OF STUDY}

Because this study was not double blind, the exercise time and work rate in exercise periods two and three were subjectively controlled by the physicians to ensure an adequate exercise duration. However, the initial exercise test was performed to a maximal workload. This explains why the duration of exercise and work rate were not different in the different pacing modes. However, other more important variables of cardiopulmonary function, such as oxygen uptake, anaerobic threshold, and end tidal pressure of carbon dioxide are independent of the amount of exercise if anaerobic threshold is reached, as it was in this study. ${ }^{21}$ Also this study related only to the short term benefits of different pacing modes. The long term effects of DDD pacing at different atrioventricular intervals are unknown.

\section{CLINICAL IMPLICATIONS}

This study suggests that pacing with short or long atrioventricular intervals is well tolerated and provided similar physiological benefits during exercise in patients with normal or reduced left ventricular function. Clearly, it is not necessary to use unusually short atrioventricular intervals to achieve additional physiological benefits at rest or during exercise. In fact, very short atrioventricular intervals (< $150 \mathrm{~ms}$ ) may produce unnecessary ventricular pacing and shorten the useful life of the battery.

We thank Dr Richard Casaburi for his review of the manuscript.

\section{References}

1 Karlof I. Haemodynamic effect of atrial triggered versus fixed rate pacing at rest and during exercise in complete heart block. Acta Med Scand 1975;197: 195-206.

2 Reiter MJ, Hindman MC. Hemodynamic effects of acute atrioventricular sequential pacing in patients with left ventricular dysfunction. Am $J$ Cardiol 1982;49:687-92.

3 Perrins EJ, Morley CA, Chan SL, Sutton R. Randomised controlled trial of physiological and ven- tricular pacing. Br Heart $J$ 1983;50:112-7.

4 Hartzler GO, Maloney JD, Curtis JJ, et al. Hemodynamic benefits of atrioventricular sequential pacing after cardiac surgery. Am J Cardiol 1977;40:232-6.

5 Benchimol A, Ellis JG, Dimond EG. Hemodynamic consequences of atrial and ventricular pacing in patients with normal and abnormal hearts. Effect of exercise at a fixed atrial and ventricular rate. $A m J M e d$ 1965;39:911-22.

6 Ausubel K, Furman S. The pacemaker syndrome. Ann Intern Med 1985;103:420-9.

7 Sutton R, Morley C, Chan SL, et al. Physiological benefits of atrial synchrony in paced patients. PACE 1983;6:327-8.

8 Haskell RJ, French WJ. Optimum AV interval in dual chamber pacemakers. $P A C E$ 1986;98:670-4.

9 Faerestrand $S, O h m$ OJ. A time-related study of the hemodynamic benefit of atrioventricular synchronous pacing evaluated by Doppler echocardiography. $P A C E$ 1985;8:838-48.

10 von Bibra $H$, Wirtzfeld A, Hall $R$, Ulm K, Blömar $H$. Mitral valve closure and left ventricular filling time in patients with VDD pacemakers. Assessment of the onset of left ventricular systole and the end of diastole. Br Heart J 1986;55:355-63.

11 Wasserman K. Determinants and detection of anaerobic threshold and consequences of exercise above it. Circulation 1987;76(suppl VI):29-39.

12 Bruning JL, Kintz BL. Computional handbook of statistics. Glenview, Illinois: Scott, Foresman, 1977:46-50.

13 Samet P, Castillo C, Bernstein WH. Hemodynamic sequelas of atrial, ventricular and sequential atrioventricular pacing in cardiac patients. Am Heart J 1966;72:725-9.

14 Kruse I, Ryden L. Comparison of physical work capacity and systolic time intervals with ventricular inhibited and atrial synchronous ventricular inhibited pacing. Br Heart J 1981;46:129-36.

15 Carleton RA, Passovoy M, Graettinger JS. The importance of the contribution and timing of left atrial systole. Clin Sci 1966;30:151-9.

16 Rahimtoola SH, Ehsani A, Sinns MZ, et al. Left atrial transport function in myocardial infarction. Importance of its booster pump function. Am J Med 1975;59:686-94.

17 Fananapazir L, Srinivas V, Bennett DH. Comparison of resting hemodynamic indices and exercise performance during atrial synchronized and asynchronous pacing. $P A C E$ 1983;6:202-9.

18 Shapland JE, MacCarter D, Tockman B, et al. Physiologic benefit of rate responsiveness. PACE 1983;6:329-32.

19 Benditt DG, Mianulli M, Fetter J, et al. Singlechamber cardiac pacing with activity-initiated chronotropic response: evaluation by cardiopulmonary exercise testing. Circulation 1987;75:184-91.

20 Humen DP, Kostuk WJ, Klein GJ. Activity-sensing, rate-responsive pacing: improvement in myocardial performance with exercise. PACE 1985;8:52-9.

21 Wasserman $\mathrm{K}$. Use of exercise in cardiopulmonary assessment of exertional dyspnea. In: Baum GL, Wolinsky E, eds. Textbook of pulmonary disease. Boston: Little, Brown, 1983:278-9. 